\title{
MAHOVNA FLORA SMREKOVŠKEGA POGORJA (KAMNIŠKO-SAVINJSKE ALPE, SLOVENIJA)
}

\author{
Andrej MARTINČIČ**
}

\section{Izvleček}

V članku avtor podaja rezultate lastnih florističnih raziskovanj mahovne flore Smrekovškega pogorja, vključuje pa tudi podatke iz literature. Mahovna flora obsega 229 vrst, od tega 173 vrst listnatih mahov (Bryophyta) in 56 vrst jetrenjakov (Marchantiophyta). Med njimi so 3 vrste nove za Slovenijo (Sciuro-hypnum oedipodium, Calypogeia integristipula, Lophozia sudetica), 20 vrst pa je v Kamniško-Savinjskih Alpah (alpsko fitogeografsko območje) omenjeno prvič. Podana je tudi fitogeografska analiza. V Rdeči seznam ogrožene mahovne flore Slovenije je vključeno 26 vrst: 13 v kategoriji »redke«, 12 v kategoriji »ranljive« in 1 v kategoriji »ogrožene« - Sphagnum compactum. Na evropskem Rdečem seznamu (ECCB 1995) so 4 vrste: Scapania verrucosa, Brachythecium geheebii, Fissidens asplenioides in Paraleucobryum sauteri.

Ključne besede: mahovna flora, Bryophyta, Marchantiophyta, Smrekov̌̌ko pogorje (Kamniško-Savinjske Alpe), horološka analiza, vrste na Rdečem seznamu.

\begin{abstract}
The study gives results on new bryophyte records of Smrekovško pogorje Mts. as well as the previous reports from the literature. In total 229 species are reported from the investigated region, 173 mosses and 56 liverworts. Among them 3 are reported for the first time for Slovenia (Sciuro-hypnum oedipodium, Calypogeia integristipula, Lophozia sudetica), 20 species are reported for the first time for the Kamniško-Savinjske Alps (Alpine phytogeographical region of Slovenia). 26 species are included in the national Red List and 4 species in the Red Data Book of European Bryophytes (ECCB 1995), in the rare (R) category: Scapania verrucosa, Brachythecium geheebii, Fissidens asplenioides and Paraleucobryum sauteri.

Key words: bryophyte flora, Bryophyta, Marchantiophyta, Smrekovško pogorje Mts. (the Kamniško-Savinjske Alps), horological analysis, red-listed species.
\end{abstract}

\section{UVOD}

Smrekovško pogorje je zaradi vulkanskih kamnin, pretežno andezita, geološka posebnost. Prav ta podlaga pa skupaj z nadmorsko višino ustvarja pogoje za posebnosti v flori predvsem višjih rastlin (Petkovšek 1945, Wraber \& Skoberne 1989). Zlasti izstopajo skalnata pobočja Komna, kjer ima nekaj vrst edino nahajališče v Sloveniji. Zato je Smrekovško pogorje proglašeno za naravni rezervat.

Flora mahov Smrekovškega pogorja je slabše poznana. Obsežnejše podatke je doslej prispeval le Wallnöfer (1888), ki je v delu »Die Laubmoose Kärntens « objavil 60 vrst listnatih mahov, pretežno s Smrekovca. Nekaj podatkov pa so prispevali tudi Wraber (1963 - det. S. Grom), Piskernik (1977 - det. A. Martinčič) in Kutnar et al. (2006 - det. A. Martinčič) v fitocenoloških prispevkih.

\section{MATERIAL IN METODE}

Kratka geografska in ekološka oznaka pogorja

Smrekovško pogorje leži na skrajnem vzhodnem delu Kamniško-Savinjskih Alp. Je značilne slemenaste oblike in se razteza v smeri vzhod-zahod. Na

* Zaloška 78a, SI-1000 Ljubljana 
vzhodu se pričenja z vrhom Smrekovec (1577 m), po katerem je dobilo pogorje ime, zaključi pa se s Presečnikovim vrhom $(1573 \mathrm{~m})$ na zahodu. Povprečna nadmorska višina je $1550 \mathrm{~m}$, le med vrhovoma Komen (1684 m) in Veliki Travnik (1637 m) je sedlo Hlipovec, kjer se sleme zniža na $1453 \mathrm{~m}$ nadmorske višine. Vrhovi so kopasti, zaobljeni, le vrh Komna je skalnat.

Smrekovško pogorje je del Kamniško-Savinjskih Alp, vendar se po nastanku in po geološkopetrografski sestavi od njih močno razlikuje. Pogorje je vulkanskega izvora, saj so bili tod v oligocenu obsežni izbruhi lave. Smrekovško pogorje je naš najvišji silikatni sklop, saj je največji del pogorja iz andezita in andezitnega tufa, le na severnem pobočju je nekaj tonalita. Na severnem pobočju Velikega Travnika je nekaj majhnih vključkov dolomita.

$\mathrm{Na}$ andezitni podlagi so nastala kisla rjava tla $\mathrm{z}$ dobro razvito humusno plastjo. Tla so razmeroma globoka, izrazito zakisana, $\mathrm{pH}$ je vedno pod 5 . Le na skalnatih pobočjih Komna je razvit ranker, močno skeleten tip tal.

Največji del Smrekovškega pogorja pokriva naravni smrekov gozd z gozdno bekico (Luzulo sylvaticae-Piceetum M. Wraber 1963), ki pa je na velikih površinah uničen zaradi paše. Obsežnejši bukovi sestoji (Luzulo-Fagetum Meusel 1937) segajo le do nadmorske višine od 1200 do $1300 \mathrm{~m}$. Omembe vreden tip vegetacije predstavljajo tudi sestoji zelene jelše (Alnetum viridis Br.-Bl. 1918). Na Komnu poraščajo skalnato severno pobočje in so primarna združba. Na nekdanjih pašnih površinah na Smrekovcu in Krnesu, ki se zaraščajo, pa nedvomno predstavljajo sekundarno razvojno stopnjo. $\mathrm{Na}$ vseh izsekanih površinah, spremenjenih v pašnike, se je razvila traviščna združba $\mathrm{z}$ volkom (Nardetum s. lat.), ki pokriva velike strnjene površine na vrhovih in slemenih pogorja. Śkornik et al. (2006) jo uvrščajo v asociacijo Homogyno alpinae-Nardetum Mráz 1956. Z opuščanjem paše se te površine polagoma zaraščajo. Zaradi nepropustne andezitne podlage so v Smrekovškem pogorju razmeroma pogosti izviri in mlake, zlasti na Krnesu in Komnu. V poletnem času nekatere mlake lahko presahnejo, namesto odprte vode je tedaj samo zamočvirjena površina.

\section{Lokalitete}

Smrekovško pogorje je v celoti locirano v dveh kvadrantih srednjeevropskega florističnega kartiranja in sicer v 9555/3 ter 9554/4 (Slika 1). Dodana je tudi lega nahajališč v UTM $(10 \times 10 \mathrm{~km})$ mreži. V seznamu vrst so imena lokalitet, na katerih smo nabirali mahovni material, navedena v skrajšani obliki (ležeče).

\section{Kvadrant 9555/3 (UTM VM94)}

1. Smrekovec, vrh (peak): 1400-1577 m, Luzulo sylvaticae-Piceetum, Nardetum s. lat.; andezitne skale, štori, gozdna in traviščna tla, izviri.

2. Kramarica, vzhodno pobočje Smrekovca: 1100 $\mathrm{m}$, Luzulo sylvaticae-Piceetum; andezitne obcestne skale, štori, gozdna tla, izviri.

3. Brezovci, severno pobočje Smrekovca: $1100 \mathrm{~m}$, Luzulo sylvaticae-Piceetum; andezitne skale, štori, gozdna tla, vlažne skale in zamočvirjena mesta ob hudourniku, na drevesni skorji.

4. Ob Vrtačnikovem potoku, na severnem vznožju grebena Krnes-Komen: 850 m; andezitne skale, gozdna tla, štori, mokre skale $\mathrm{v}$ potoku in zamočvirjena mesta (UTM VM84).

5. Kugovnik, na južnem pobočju grebena Smrekovec-Krnes: $1250 \mathrm{~m}$; andezitne skale, štori, gozdna tla, mokre skale $\mathrm{v}$ potoku.

6. Črni vrh pri Smrekovcu: $1250 \mathrm{~m}$, Luzulo sylvaticae-Piceetum; andezitne skale, štori, gozdna tla, obcestna brežina.

7. Krnes, vrh: 1500-1600 m, Alnetum viridis, Nardetum s. lat.; zamočvirjeni predeli, mlake, traviščna tla, andezitne skale.

8. Krnes, južno pobočje: $1400 \mathrm{~m}$, Luzulo sylvaticae-Piceetum; štori, gozdna tla, zamočvirjeni predeli, andezitne skale.

9. Ramšak pod Krnesom: 1300 m, Luzulo sylvaticaePiceetum; gozdna tla, štori.

10. Komen, vrh: 1600-1684 m, Alnetum viridis, Nardetum s. lat.; andezitne skale, skalne razpoke, traviščna tla (UTM VM84).

11. Mrzle vode, na severnem pobočju Komna: 1150 m, Luzulo-Fagetum; andezitne skale, gozdna tla, štori (UTM VM84).

12. Kolarica, na južnem pobočju Komna: 1480 m, Luzulo-Fagetum, andezitni groh; Kutnar et al. 2006, det. A. Martinčič (UTM VM84).

\section{Kvadrant 9554/4 (UTM VM84)}

13. Dolina reke Bistre pod Petelinjekom: $850 \mathrm{~m}$; vlažne andezitne skale, močvirna mesta, štori, skorja dreves. 


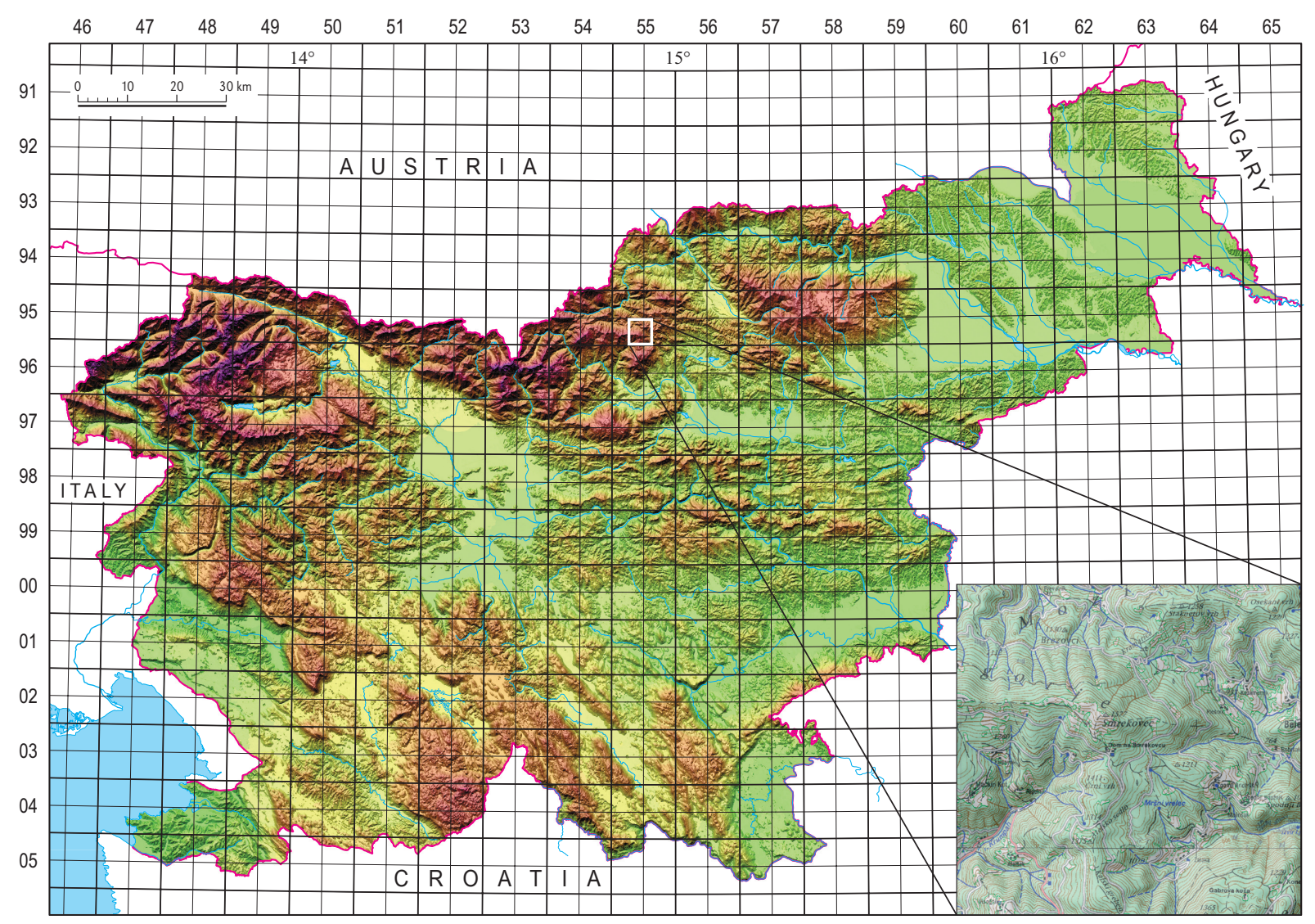

Slika 1: Lega preiskovanega območja

Figure 1: Location of the investigated area

14. Dolina reke Bistre pod Brzounikom: 750 m; vlažne andezitne skale, močvirna mesta, štori, skorja dreves.

15. Dolina reke Bistre pod Pistotnikom: 800 m; vlažne andezitne skale, močvirna mesta, štori, skorja dreves.

16. Veliki Travnik: 1500-1673 m, Luzulo sylvaticae-Piceetum; andezitne skale, štori, gozdna tla.

17. Presečnikov vrh: Luzulo sylvaticae-Piceetum, 1400 m; andezitne skale, štori, gozdna tla.

\section{Metode}

V letih od 1987 do 2007 je avtor na celotnem območju Smrekovškega pogorja nabral obsežen mahovni material, ki daje dokaj popolno floristično in fitogeografsko podobo predelov od 1000 m nadmorske višine do vrhov. Mahovi so bili nabrani v vseh pomembnih habitatih tega območja, v gozdovih, na traviščih, na skalovju, na zamočvirjenih mestih in v izvirih. Pomemben habitat, zlasti v gozdovih, so tudi obcestne brežine. Pri nabiranju smo upoštevali vse substrate, kot na primer gozdna in traviščna tla, skale, razpadajoč les in drevesno skorjo. Mahovni material je shranjen v herbariju Oddelka za biologijo Biotehniške fakultete, Univerze v Ljubljani (LJU).

Zaradi popolnosti prikaza so v delu upoštevani vsi doslej znani podatki, tako literaturni kakor tudi lastni. V nomenklaturi in taksonomiji smo sledili delu Schumacker \& Ván̆a (2005) za jetrenjake (Marchantiophyta) ter delu Hill et al. (2006) za listnate mahove (Bryophyta).

\section{REZULTATI IN RAZPRAVA}

\section{Floristični rezultati}

Mahovna flora Smrekovškega pogorja obsega 229 vrst, od tega 173 vrst listnatih mahov (Bryophyta) in 56 vrst jetrenjakov (Marchantiophyta). Pri listnatih mahovih predstavlja to število $28 \%$ trenutno ugo- 
tovljenih vrst v Sloveniji (Martinčič 2003), ter $43 \%$ vrst na območju Kamniško-Savinjskih Alp, kamor uvrščamo Smrekovško pogorje. Stevilo ugotovljenih vrst jetrenjakov pa predstavlja kar $35 \%$ trenutno v Sloveniji poznanih vrst (Sabovljević \& Natcheva 2006). Te številke pričajo o veliki pestrosti mahovne flore v obravnavanem območju. Največji del vrst je bil ugotovljen v kvadrantu $9555 / 3$ in sicer 164 vrst listnatih mahov in 53 vrst jetrenjakov. $\mathrm{S}$ tem se uvršča med 3 kvadrante v Sloveniji z najbogatejšo mahovno floro (kvadrant 9547/4 - 248 vrst, 0049/1 - 231 vrsta). Za Slovenijo smo ugotovili 3 nove vrste in sicer Sciuro-hypnum oedipodium, Calypogeia integristipula in Lophozia sudetica. Za KamniškoSavinjske Alpe, ki so del alpskega fitogeografskega območja Slovenije, so poleg naštetih nove še naslednje vrste: Chiloscyphus coadunatus var. coadunatus, Jamesoniella autumnalis, Jungermannia obovata, Marchantia polymorpha subsp. polymorpha, Ptilidium pulcherrimum med jetrenjaki in Brachythecium geheebii, B. mildeanum, Bryum schleicheri var. latifolium, Grimmia trichophylla, Hygrohypnum duriusculum, Hypnum andoi, Mnium lycopodioides, Mnium spinulosum, Plagiothecium succulentum, Racomitrium affine, Sphagnum russowii med listnatimi mahovi. Veliko vrstno pestrost izpopolnjujejo še vrste, ki imajo v KamniškoSavinjskih Alpah edino nahajališče v Smrekovškem pogorju, vendar smo jih, brez navedbe točnega nahajališča, upoštevali že v objavi iz pred nekaj let (Martinčič 2003): Entosthodon muehlenbergii, Philonotis caespitosa, P. seriata, Plagiothecium curvifolium, Polytrichastrum longisetum, Polytrichum strictum, Racomitrium fasciculare, $R$. lanuginosum, Warnstorfia exannulata in $W$. fluitans.

Lophozia sudetica: prvi navajajo za Slovenijo takson s tem imenom Düll et al. (1999). Isti podatek povzemajo zatem Söderström et al. (2002), Sabovljević \& Natcheva (2006) in Ros et al. (2007), vendar prva navedba temelji na napačnem razumevanju sinonimike. V primarni literaturi (Loitlesberger 1905, Breidler 1894, Robič 1893, Glowacki 1908, 1910, 1912, 1913, Grom 1969) ne najdemo podatka $\mathrm{s}$ tem imenom, temveč le navedbe vrst Jungermannia muelleri Nees, Lophozia muelleri (Nees) Dum., Jungermannia alpestris Schleich. ter Lophozia alpestris (Schleich.) A. Evans. Kljub določeni zmedi, ki še vedno vlada v nomenklaturi in taksonomiji, pa velja (prim. Grolle \& Long 2000, Damsholt 2002, Schumacher \& Váňa 2005), da navedeni taksoni nimajo nobene nomenklatorične povezave $\mathrm{z}$ vrsto Lophozia sudetica. Do napake je torej prišlo v Düll et al. (1999), kar potrjuje dodani sinonim (L. alpestris
(Schleich.) Ev.). Vrsta L. sudetica (Nees ex Huebener) Grolle (syn: L. alpestris auct. non (Schleich.) A. Evans) je torej nova vrsta za slovensko mahovno floro z dvema nahajališčema na Smrekovškem pogorju.

Calypogeia integristipula: borealno-temperatna vrsta s težiščem uspevanja v severni polovici Evrope, proti jugu postaja vse redkejša. Na Balkanskem polotoku je znana samo iz Srbije (Sabovljević \& Natcheva 2006). Nahajališča v Sloveniji se navezujejo na širši srednjeevropski del areala. Poleg nahajališč v Smrekovškem pogorju uspeva vrsta še na več mestih v alpskem fitogeografskem območju Slovenije (neobjavljeno), vedno v montanskem pasu, na gozdni zemlji, na drevesnih štorih, pa tudi na skalah.

Sciuro-hypnum oedipodium: temperatni element, raztreseno razširjen od južne Skandinavije preko srednje Evrope, vendar manjka v atlantsko-mediteranskih predelih. Zaradi podobnosti z vrstama B. rutabulum in B. starkei je marsikje prezrt. To je najbrž vzrok, da se južno od Slovenije pojavlja šele v Prokletijah (Martinčič 2006) ter v Romuniji in Grčiji (Sabovljević et al. 2008, v tisku). V Smrekovškem pogorju je bil najden na dveh nahajališčih ter na vrhu Altarna peč v neposredni bližini, v gozdnih sestojih na vlažnih tleh in na štorih.

\section{Seznam taksonov}

$\mathrm{Z} *$ so označene »ranljive« $(\mathrm{V})$ vrste iz Rdečega seznama, $\mathrm{z} * *$ so označene »redke« $(\mathrm{R})$ vrste.

\section{Bryophyta - listnati mahovi}

Abietinella abietina (Hedw.) M. Fleisch. var. abietina: $u b i k v-S m r e k o v e c$ (Piskernik 1977).

Amblystegium subtile (Hedw.) Schimp.: temp-subkont - Krnes, juž. pob.; pod Pistotnikom.

**Amphidium lapponicum (Hedw.) Schimp.: subarktsubalp-Smrekovec, na grebenu, $1460 \mathrm{~m}$ (Wallnöfer 1888).

Amphidium mougeotii (Schimp.) Schimp.: bor-mont - Smrekovec (Wallnöfer 1888); Kramarica; Kugovnik; Komen (leg. B. Druškovič); Presečnikov vrh.

**Andreaea rupestris Hedw. var. rupestris: bor-mont - Smrekovec (Wallnöfer 1888); Smrekovec; Veliki Travnik. 
Anomodon attenuatus (Hedw.) Hueben.: subtemp - Smrekovec.

Anomodon rugelii (Müll.-Hal.) Keissl.: temp-subkont - Smrekovec (M. Wraber 1963, det. S. Grom).

Anomodon viticulosus (Hedw.) Hook. \& Taylor: bortemp-Smrekovec (M. Wraber 1963).

Antitrichia curtipendula (Hedw.) Brid.: bor-temp Smrekovec, sev. pob.

Atrichum tenellum (Röhl.) Bruch \& Schimp.: bortemp-Krnes.

Atrichum undulatum (Hedw.) P. Beauv.: bor-temp Smrekovec (Wallnöfer 1888, M. Wraber 1963); Smrekovec, vzh. pob.

Aulacomnium palustre (Hedw.) Schwägr.: subbor - Krnes.

Bartramia halleriana Hedw.: bor-mont - Kramarica; Smrekovec, vzh. pob.; Presečnikov vrh; pod Brzovnikom.

Bartramia ithyphylla Brid.: bor-mont - Smrekovec (Wallnöfer 1888).

Bartramia pomiformis Hedw.: bor-temp - Smrekovec (leg. F. Sušnik); Kugovnik.

**Blindia acuta (Hedw.) Bruch \& Schimp.: bor-mont - Presečnikov vrh.

Brachytheciastrum velutinum (Hedw.) Ignatov \& Huttunen: temp - Črni vrh; Kramarica; Brezovci; Kugovnik; Krnes, juž. pob.; pod Brzovnikom.

Brachythecium campestre (Müll.-Hal.) Schimp.: tempsubkont-Brezovci; Kugovnik; pod Brzovnikom.

Brachythecium geheebii Milde: temp-subkont-Kugovnik.

Brachythecium mildeanum (Schimp.) Schimp.: temp - Kramarica.

Brachythecium rivulare Schimp.: bor-temp - Smrekovec; Kugovnik; Vrtačnikov potok; Krnes.

Brachythecium rutabulum (Hedw.) Schimp.: temp - Smrekovec, juž. pob.; pod Brzovnikom.

Brachythecium tommasinii (Sendtn. ex Boulay) Ignatov \& Huttunen: temp-subkont - pod Pistotnikom.

Bryoerythrophyllum recurvirostrum (Hedw.) P. C. Chen: bor-temp - Kramarica; Smrekovec (leg. F. Sušnik); Smrekovec (Wallnöfer 1888); Krnes (Wallnöfer 1888); Komen (Wallnöfer 1888).

Bryum argenteum Hedw. var. lanatum (P. Beauv.) Hampe: ubikv-Kugovnik.

Bryum capillare Hedw.: ubikv-Kugovnik.

Bryum pseudotriquetrum (Hedw.) P. Gaertn. et al. var. pseudotriquetrum: ubikv-Kugovnik.

Bryum schleicheri DC. var. latifolium (Schwägr.) Bruch \& Schimp.: bor-mont-Krnes, juž. pob.

Calliergonella cuspidata (Hedw.) Loeske: temp Smrekovec, vzh. pob.
Campylium protensum (Brid.) Kindb.: bor-temp Smrekovec, sev. pob.; pod Brzovnikom.

Ceratodon purpureus (Hedw.) Brid. subsp. purpureus: ubikv-Kramarica; Smrekovec; Kugovnik.

Cirriphyllum crassinervium (Taylor) Loeske \& M. Fleisch.: temp - Kramarica; ob Vrtačnikovem potoku.

Cirriphyllum piliferum (Hedw.) Grout: bor-temp-Klipovčnik v dolini Bistre (Wallnöfer 1888); ob Vrtačnikovem potoku.

Cratoneuron filicinum (Hedw.) Spruce: ubikv-Smrekovec; Krnes.

Ctenidium molluscum (Hedw.) Mitt.: bor-temp - Kramarica (Wallnöfer 1888); Brezovci; pod Pistotnikom.

Ctenidium molluscum (Hedw.) Mitt. var. condensatum (Schimp.) Brid.: temp - ob Vrtačnikovem poto$\mathrm{ku}$.

Cynodontium polycarpon (Hedw.) Schimp.: bor-mont - Kramarica; Kugovnik; Veliki Travnik.

**Cynodontium strumiferum (Hedw.) Lindb.: bormont-Smrekovec (Wallnöfer 1888); Smrekovec (leg. F. Sušnik); Kugovnik; Presečnikov vrh.

Dichodontium palustre (Dicks.) M. Stech.: bor-mont - Smrekovec (pri Krumpaški planini); Kugovnik; Krnes.

Dichodontium pellucidum (Hedw.) Schimp.: bor-mont - Smrekovec (Wallnöfer 1888); Smrekovec, vzh. pob.; ob Vrtačnikovem potoku; pod Brzovnikom; Presečnikov vrh.

Dicranella heteromalla (Hedw.) Schimp.: bor-temp - Črni vrh; Kramarica; Smrekovec (Wallnöfer 1888); Smrekovec; Krnes; Veliki Travnik.

Dicranella rufescens (Dicks.) Schimp.: bor-temp Smrekovec, vzh. pob.

Dicranella subulata (Hedw.) Schimp.: bor-mont Smrekovec (Wallnöfer 1888).

Dicranella varia (Hedw.) Schimp.: ubikv-Črni vrh; Smrekovec, sev. pob.

Dicranodontium denudatum (Hedw.) E. Britton var. denudatum: bor-mont - Smrekovec (pri planinskem domu).

**Dicranoweisia crispula (Hedw.) Milde: bor-mont - Smrekovec (Wallnöfer 1888); Krnes, juž. pob.; Komen; Veliki Travnik.

Dicranum montanum Hedw.: bor-temp - Smrekovec.

Dicranum polysetum Sw.: bor-mont-Smrekovec.

Dicranum scoparium Hedw.: subbor - Črni vrh; Kramarica; Smrekovec (Wallnöfer 1888, Piskernik 1977); Kugovnik; Komen; pod Brzovnikom; Veliki Travnik; Presečnikov vrh.

Didymodon acutus (Brid.) K. Saito: merid-temp - pod Pistotnikom. 
Didymodon fallax (Hedw.) R. H. Zander: ubikv- Kugovnik.

Diphyscium foliosum (Hedw.) D. Mohr: bor-temp - Smrekovec (Wallnöfer 1888).

Distichium capillaceum (Hedw.) Bruch \& Schimp.: bor-mont - Smrekovec (Wallnöfer 1888); Smrekovec; Komen; Veliki Travnik.

Ditrichum heteromallum (Hedw.) E. Britton: bor-temp - Krnes (Wallnöfer 1888).

Drepanocladus aduncus (Hedw.) Warnst.: bor-temp - Kramarica (Wallnöfer 1888).

Encalypta streptocarpa Hedw.: bor-temp-Smrekovec, sev. pob.

Entosthodon muehlenbergii (Turner) Fife: merid-temp - Komen.

Eurhynchium angustirete (Broth.) T. J. Kop.: tempsubkont-Smrekovec (Piskernik 1977); Smrekovec, sev. pob.; Mrzle vode.

Fissidens adianthoides Hedw.: bor-temp - Smrekovec (Wallnöfer 1888).

Fissidens dubius P. Beauv.: temp - Smrekovec; pod Brzovnikom.

Fissidens dubius P. Beauv. var. mucronatus Breidler - Kramarica; Komen.

Fontinalis antipyretica Hedw. subsp. antipyretica: bortemp-Smrekovec (pri Krumpaški planini).

Funaria hygrometrica Hedw.: ubikv - Smrekovec (Wallnöfer 1888).

Grimmia hartmanii Schimp.: subbor - Smrekovec; Mrzle vode; Vk Travnik.

Grimmia pulvinata (Hedw.) Sm.: merid-temp-Smrekovec (M. Wraber 1963); Smrekovec; pod Pistotnikom.

* Grimmia trichophylla Grev.: temp-Komen.

Hedwigia ciliata (Hedw.) P. Beauv. var. ciliata: bortemp - Smrekovec (Wallnöfer 1888); Kugovnik; Komen.

Herzogiella seligeri (Brid.) Z. Iwats.: subtemp - Kramarica (Wallnöfer 1888); Smrekovec (Wallnöfer 1888); Smrekovec; ob Vrtačnikovem potoku; Ramšak; Mrzle vode; pod Brzovnikom.

Heterocladium dimorphum (Brid.) Schimp.: bor-mont - Kramarica (Wallnöfer 1888); Kolarica (Kutnar et al. 2006 - det. A. Martinčič).

Heterocladium heteropterum (Brid.) Schimp.: tempsubatl-ob Vrtačnikovem potoku.

Homalothecium phillipeanum (Spruce) Schimp.: temp-subkont (srednjeewr) - Smrekovec, sev. pob.; pod Pistotnikom.

Homalothecium sericeum (Hedw.) Schimp.: meridtemp-Smrekovec, vzh. pob.

*Hygrohypnum duriusculum (De Not.) D. W. Jamieson: subarkt-subalp-Kugovnik.
Hygrohypnum luridum (Hedw.) Jenn.: bor-temp Smrekovec (Wallnöfer 1888); Brezovci.

Hygrohypnum luridum var. subsphaericarpon (Schleich. ex Brid.) C. Jens.: bor-mont - pod Brzovnikom.

Hylocomiastrum umbratum (Hedw.) M. Fleisch.: bormont-Krnes (M. Wraber 1963).

Hylocomium splendens (Hedw.) Schimp.: subbor Kramarica; Smrekovec (Piskernik 1977); Smrekovec; pod Pistotnikom.

Hypnum andoi A. J. E. Sm.: temp-subatl-Kramarica; Smrekovec; ob Vrtačnikovem potoku.

Hypnum cupressiforme Hedw. var. cupressiforme: ubikv - Kramarica; Smrekovec; Kugovnik; Krnes, juž. pob.; Mrzle vode; pod Brzovnikom.

Hypnum cupressiforme Hedw. var. filiforme Brid.: temp-Kugovnik; pod Pistotnikom.

Hypnum cupressiforme Hedw. var. lacunosum Brid.: temp-Smrekovec; Veliki Travnik.

Hypnum cupressiforme Hedw. var. resupinatum (Taylor) Schimp. - Smrekovec; pod Pistotnikom.

Hypnum jutlandicum Holmen \& E. Warncke: tempsubatl-Kugovnik.

Isothecium alopecuroides (Lam. ex Dubois) Isov.: bortemp - Smrekovec (Wallnöfer 1888, M. Wraber 1963, M. Piskernik 1977); Smrekovec; Kugovnik; Krnes (M. Wraber 1963); Krnes, juž. pob.; Mrzle vode; Veliki Travnik.

Kindbergia praelonga (Hedw.) Ochyra: temp-Smrekovec.

Leucobryum glaucum (Hedw.) Ångstr.: temp - Smrekovec (Wallnöfer 1888, Piskernik 1977); Krnes; Komen; Veliki Travnik.

Leucodon sciuroides (Hedw.) Schwägr. var. sciuroides: subtemp - Smrekovec (Wallnöfer 1888); Smrekovec, sev. pob.; Veliki Travnik.

Loeskeobryum brevirostre (Brid.) M. Fleisch.: temp - Smrekovec (Wallnöfer 1888).

Mnium lycopodioides Schwägr.: bor-mont - Smrekovec, vzh. pob.

Mnium marginatum (Dicks.) P. Beauv.: bor-temp Smrekovec (Wallnöfer 1888); ob Vrtačnikovem potoku.

Mnium spinulosum Bruch \& Schimp.: temp-subkont (srednjeevr) - pod Pistotnikom.

Mnium stellare Hedw.: bor-temp - Smrekovec, vzh. pob.; pod Pistotnikom.

Mnium thomsonii Schimp.: bor-mont-pod Petelinjekom; pod Brzovnikom.

Neckera complanata (Hedw.) Huebener: bor-temp - Smrekovec (Wallnöfer 1888).

Neckera crispa Hedw.: temp - Smrekovec (Wallnöfer 1888); Smrekovec, vzh. pob. 
**Oligotrichum hercynicum (Hedw.) Lam \& DC.: subarkt-subalp - Smrekovec, vzh. pob.; Komen; Vk, Travnik.

Orthothecium rufescens (Brid.) Bruch \& Schimp.: bormont-Smrekovec (Wallnöfer 1888).

Orthotrichum cupulatum Hoffm. ex Brid.: temp Smrekovec, sev. pob.

Orthotrichum pumilum Sw.: temp - Kramarica (Wallnöfer 1888).

Orthotrichum speciosum Nees: bor-temp - Smrekovec, sev. pob.; ob Vrtačnikovem potoku; pod Brzovnikom.

Orthotrichum stramineum Hornsch. ex Brid.: temp - ob Vrtačnikovem potoku.

Oxyrrhynchium hians (Hedw.) Loeske: temp - Smrekovec, sev. pob.; Kugovnik; pod Brzovnikom.

Oxyrrhynchium schleicheri (R. Hedw.) Röll: meridtemp-Smrekovec, sev. pob.

Oxyrrhynchium speciosum (Brid.) Warnst.: temp Brezovci.

Palustriella commutata (Hedw.) Ochyra: bor-temp - Smrekovec (Wallnöfer 1888); Krnes.

Paraleucobryum longifolium (Hedw.) Loeske var. longifolium: bor-mont - Kramarica; Smrekovec (Wallnöfer 1888); Smrekovec; ob Vrtačnikovem potoku; Ramšak; pod Petelinjekom.

Paraleucobryum sauteri (Bruch \& Schimp.) Loeske: temp-subkont-Veliki Travnik.

* Philonotis arnellii Husn.: temp - Kugovnik.

*Philonotis caespitosa Jur.: bor-temp - Smrekovec (Wallnöfer 1888); Krnes.

Philonotis fontana (Hedw.) Brid.: bor-temp - Kugovnik; Krnes, juž. pob.; Presečnikov vrh.

*Philonotis seriata Mitt.: subarkt-subalp-Krnes.

*Philonotis tomentella Molendo: arkt-alp - Krnes; Presečnikov vrh.

Plagiomnium affine (Blandow ex Funck) T. J. Kop.: temp-Kugovnik.

Plagiomnium cuspidatum (Hedw.) T. J. Kop.: bor-temp - Smrekovec (Wallnöfer 1888).

Plagiomnium elatum (Bruch \& Schimp.) T. J. Kop.: bor-temp-Smrekovec, vzh. pob.; Mrzle vode.

Plagiomnium medium (Bruch \& Schimp.) T. J. Kop.: bor-mont - Smrekovec, vzh. pob.; Kugovnik; ob Vrtačnikovem potoku; pod Petelinjekom.

Plagiomnium rostratum (Schrad.) T. J. Kop.: bor-temp - Smrekovec, vzh. pob.; ob Vrtačnikovem potoku; Brezovci; pod Petelinjekom.

Plagiomnium undulatum (Hedw.) T. J. Kop.: temp Smrekovec, vzh. pob.; ob Vrtačnikovem potoku.

Plagiopus oederianus (Sw.) H. A. Crum \& L. E. Anderson: bor-mont-Smrekovec (Wallnöfer 1888); pod Pistotnikom; Veliki Travnik.
Plagiothecium cavifolium (Brid.) Z. Iwats.: bor-montSmrekovec, vzh pob.; ob Vrtačnikovem potoku.

**Plagiothecium curvifolium Schlieph. ex Limpr.: temp - Ramšak (leg. M. Zupančič, Kutnar et al. 2006); Kolarica (Kutnar et al. 2006); Veliki Travnik.

Plagiothecium denticulatum (Hedw.) Schimp.: bortemp - Črni vrh; Smrekovec (Wallnöfer 1888, Piskernik 1977).

Plagiothecium laetum Schimp.: bor-mont - Kramarica; Smrekovec; Kugovnik; Komen; pod Brzovnikom; Veliki Travnik.

Plagiothecium nemorale (Mitt.) A. Jaeger: temp Smrekovec (Wallnöfer 1888, Piskernik 1977); Smrekovec; pod Petelinjekom.

Plagiothecium platyphyllum Mönk.: bor-mont - Smrekovec.

**Plagiothecium succulentum (Wilson) Lindb.: bortemp - Smrekovec, vzh. pob.; ob Vrtačnikovem potoku.

Plagiothecium undulatum (Hedw.) Schimp.: bor-temp - Smrekovec (Wallnöfer 1888); Smrekovec, vzh. pob.; Krnes, juž. pob.

Platyhypnidium riparioides (Hedw.) Dixon: temp - Smrekovec; Brezovci; Vrtačnikov potok; reka Bistra pod Brzovnikom.

Pleurozium schreberi (Willd. ex Brid.) Mitt.: bor-temp - Smrekovec (Wallnöfer 1888); Smrekovec; Krnes; Mrzle vode; pod Pistotnikom.

Pogonatum aloides (Hedw.) P. Beauv.: bor-temp - Črni vrh; Smrekovec (Wallnöfer 1888, M. Wraber 1963); Smrekovec, vzh. pob.; Krnes (M. Wraber 1963); Krnes; Komen; Veliki Travnik.

Pogonatum urnigerum (Hedw.) P. Beauv.: bor-montČrni vrh; Kramarica; Komen (Martinčič 1977); pod Pistotnikom; Veliki Travnik; Presečnikov vrh.

Pohlia elongata Hedw.: bor-mont-Smrekovec (Wallnöfer 1888).

Pohlia wahlenbergii (F. Weber \& D. Mohr) A. L. Andrews: subbor-Smrekovec (Wallnöfer 1888).

Polytrichastrum alpinum (Hedw.) G. L. Sm.: subarktsubalp-Smrekovec (Wallnöfer 1888); Krnes.

Polytrichastrum formosum (Hedw.) G. L. Sm.: bortemp-Črni vrh; Kramarica; Smrekovec; Komen; pod Petelinjekom; Veliki Travnik.

Polytrichastrum longisetum (Sw. ex Brid.) G. L. Sm.: bor-temp - Kramarica (Wallnöfer 1888); Smrekovec (Wallnöfer 1888); Krnes (M. Wraber 1963).

Polytrichum commune Hedw. subsp. commune: subbor - Kramarica; Smrekovec (Wallnöfer 1888); Smrekovec; Krnes; Komen (leg. B. Druškovič); Veliki Travnik; Presečnikov vrh. 
Polytrichum juniperinum Hedw.: bor-temp - Komen (leg. B. Druškovič); Presečnikov vrh.

Polytrichum piliferum Hedw.: bor-temp - Krnes; Komen.

Polytrichum strictum Menzies ex Brid.: bor-temp- Krnes; Komen (leg. B. Druškovič).

Pseudotaxiphyllum elegans (Brid.) Z. Iwats.: subarktsubalp - Kramarica; Smrekovec (Piskernik 1977); Smrekovec; ob Vrtačnikovem potoku; Krnes, juž. pob.; pod Petelinjekom; Veliki Travnik.

Pterigynandrum filiforme Hedw.: bor-mont - Smrekovec; pod Pistotnikom.

Pylaisia polyantha (Hedw.) Schimp.: subtemp-Smrekovec.

Racomitrium affine (F. Weber \& D. Mohr) Lindb.: temp-Presečnikov vrh.

* Racomitrium aquaticum (Brid. ex Schrad.) Brid.: temp - Smrekovec (Wallnöfer 1888); Smrekovec; Brezovci; Komen; Presečnikov vrh.

Racomitrium canescens (Hedw.) Brid.: bor-mont Smrekovec (M. Wraber 1963); Komen.

*Racomitrium fasciculare (Hedw.) Brid.: bor-temp - Komen.

Racomitrum heterostichum (Hedw.) Brid.: bor-temp - Kramarica; Smrekovec (Wallnöfer 1888); Smrekovec (leg. M. Zupančič); Komen; Veliki Travnik; Presečnikov vrh.

**Racomitrium lanuginosum (Hedw.) Brid.: bor-mont - Komen.

**Racomitrium sudeticum (Funck) Bruch \& Schimp.: subarkt-subalp - Smrekovec (Wallnöfer 1888); Komen, vrh.

Rhizomnium punctatum (Hedw.) T. J. Kop.: bor-temp - Smrekovec (Wallnöfer 1888); Smrekovec; Brezovci; Krnes; ob Vrtačnikovem potoku; Presečnikov vrh.

Rhynchostegiella tenella (Dicks.) Limpr.: merid-temp - Kramarica.

Rhynchostegium murale (Hedw.) Schimp.: temp-ob Vrtačnikovem potoku.

Rhytidiadelphus loreus (Hedw.) Warnst.: bor-temp - Kramarice; Smrekovec (Wallnöfer 1888); Komen; Veliki Travnik.

Rhytidiadelphus squarrosus (Hedw.) Warnst.: bortemp - Smrekovec (Wallnöfer 1888); pod Petelinjekom; pod Pistotnikom.

Rhytidiadelphus triquetrus (Hedw.) Warnst.: bor-temp - Smrekovec (Wallnöfer 1888); Smrekovec, sev. pob.; Komen; pod Pistotnikom.

Rhytidium rugosum (Hedw.) Kindb.: bor-temp-Smrekovec (Wallnöfer 1888).
Sanionia uncinata (Hedw.) Loeske: bor-mont - Kramarica (Wallnöfer 1888); Kramarica; Smrekovec, vzh. pob.; Krnes, juž. pob.; Komen (leg. T. Planina); pod Petelinjekom.

Sciuro-hypnum oedipodium (Mitt.) Ignatov \& Huttunen: temp-Smrekovec, vzh. pob.; pod Brzovnikom.

Sciuro-hypnum plumosum (Hedw.) Ignatov \& Huttunen: bor-temp - Kramarica; ob Vrtačnikovem potoku; pod Petelinjekom.

Sciuro-hypnum populeum (Hedw.) Ignatov \& Huttunen: temp - pod Pistotnikom.

Sciuro-hypnum starkei (Brid.) Ignatov \& Huttunen: bor-mont - Črni vrh; Smrekovec, vzh. pob.; Veliki Travnik.

Sphagnum capillifolium (Ehrh.) Hedw.: bor-temp - Smrekovec, vzh. pob.; Krnes; Komen.

Sphagnum compactum Lam. \& DC.: bor-temp - Komen.

Sphagnum girgensohnii Russow: bor-mont - Komen (leg. B. Druškovič); Veliki Travnik.

Sphagnum palustre L.: bor-temp - pri Kugovniku.

Sphagnum quinquefarium (Braithw.) Warnst.: bormont-Presečnikov vrh.

Sphagnum russowii Warnst.: bor-mont - Komen; Veliki Travnik.

Sphagnum subnitens Russow \& Warnst.: bor-temp-Komen; Veliki Travnik.

Taxiphyllum wisgrillii (Garov.) Wijk \& Margad.: temp - Smrekovec (Piskernik 1977); Krnes (M. Wraber 1963).

Tetraphis pellucida Hedw.: bor-temp-Črni vrh; Smrekovec (Wallnöfer 1888); Smrekovec; ob Vrtačnikovem potoku; Krnes, juž. pob.; Komen (leg. et det. T. Planina); pod Brzovnikom.

Thamnobryum alopecurum (Hedw.) Nieuvl.: temp - Smrekovec.

Thuidium delicatulum (Hedw.) Schimp.: temp - Kramarica (Wallnöfer 1888); pod Pistotnikom.

Thuidium tamariscinum (Hedw.) Schimp.: temp Smrekovec (Wallnöfer 1888); Brezovci.

Tortella tortuosa (Hedw.) Limpr.: bor-temp - Kramarica; pri Kugovniku; Komen; pod Pistotnikom.

Trichodon cylindricus (Hedw.) Schimp.: bor-temp - Smrekovec (Wallnöfer 1888).

Ulota crispa (Hedw.) Brid.: temp-ob Vrtačnikovem potoku; pod Pistotnikom.

Warnstorfia exannulata (Schimp.) Loeske: bor-temp - Krnes.

*Warnstorfia fluitans (Hedw.) Loeske: bor-temp- Krnes. 
Marchantiophyta-jetrenjaki

Anastrophyllum michauxii (F. Weber) H. Buch: subarkt-subalp-Kramarica.

Anastrophyllum minutum (Schreb.) R. M. Schust. var. weberi (Mart.) Kartt.: bor-mont - Komen.

*Aneura pinguis (L.) Dumort.: subbor-Smrekovec.

Apometzgeria pubescens (Schrank.) Kuwah.: bor-mont - pod Brzovnikom.

Bazzania trilobata (L.) Gray: subbor - ob Vrtačnikovem potoku; pod Petelinjekom.

Blepharostoma trichophyllum (L.) Dumort. var. trichophyllum: subbor - Smrekovec, vzh. pob.; Brezovc; ob Vrtačnikovem potoku; pod Brzovnikom; Veliki Travnik.

Calypogeia azurea Stotler \& Crotz: subbor- Črni vrh; Smrekovec, vzh. pob.; pri Kugovniku; ob Vrtačnikovem potoku; Krnes, juž. pob.; Komen, vrh; Mrzle vode; pod Brzovnikom.

**alypogeia integristipula Steph.: subtemp - Kramarica; pri Kugovniku; Smrekovec; Krnes, juž. pob.

Calypogeia muelleriana (Schiffn.) Müll.-Hal.: subbor - Smrekovec; ob Vrtačnikovem potoku.

**Calypogeia neesiana (C. Massal. \& Carestia) Müll. Frib.: bor-mont-Smrekovec, vzh. pob.

Cephalozia bicuspidata (L.) Dumort. var. bicuspidata: bor-temp-Smrekovec; ob Vrtačnikovem potoku; Krnes, juž. pob.; Komen; Veliki Travnik.

Cephalozia bicuspidata (L.) Dumort. var. lammersiana (Hueben.) Breidl.: bor-temp - Krnes, juž. pob.

Cephalozia leucantha Spruce: bor-mont - Veliki Travnik.

Cephalozia lunulifolia (Dumort.) Dumort.: bor-mont - Smrekovec, vzh. pob.

Chiloscyphus coadunatus (Sw.) J. J. Engel \& R. M. Schust. var. coadunatus: temp - Črni vrh; Smrekovec, sev. pob.; Krnes, juž. pob.; pod Petelinjekom.

Chiloscyphus coadunatus (Sw.) J. J. Engel \& R. M. Schust. var. rivularis Loeske: temp-Smrekovec, vzh. pob.; pod Brzovnikom.

Chiloscyphus polyanthos (L.) Corda var. polyanthos: subbor-Smrekovec; pri Kugovniku; Krnes; pod Brzovnikom.

Chiloscyphus polyanthos (L.) Corda var. rivularis (Schrad.) Gottsche et al.: subbor - Smrekovec, vzh. pob.; ob Vrtačnikovem potoku.

Chiloscyphus profundus (Nees) J. J. Engel \& R. M. Schust.: temp - Smrekovec; Ramšak (Kutnar et al. 2006); ob Vrtačnikovem potoku; Krnes; pod Pistotnikom.
Conocephalum conicum (L.) Dumort.: subbor-Smrekovec, vzh. pob.; Brezovc; pri Kugovniku; pod Brzovnikom.

Diplophyllum albicans (L.) Dumort.: bor-temp - Kramarica; pri Kugovniku; Komen; Presečnikov vrh.

Diplophyllum obtusifolium (Hook.) Dumort.: bor-temp - Kolarica (leg. L. Kutnar).

Diplophyllum taxifolium (Wahlenb.) Dumort.: subarkt-subalp - Crni vrh; Krnes, juž. pob.

Frullania dilatata (L.) Dumort.: merid-temp-Smrekovec, sev. pob.; ob Vrtačnikovem potoku; pod Pistotnikom.

Jamesoniella autumnalis (DC.) Steph.: subbor - Krnes, juž. pob.

Jungermannia atrovirens Dumort.: bor-temp - pri Kugovniku.

Jungermannia leiantha Grolle: bor-mont - ob Vrtačnikovem potoku; pod Brzovnikom.

Jungermannia obovata Nees: bor-mont-Brezovci.

Jungermannia pumila With.: subbor - pod Brzovnikom.

Jungermannia sphaerocarpa Hook.: bor-mont - pri Kugovniku.

Lejeunea cavifolia (Ehrh.) Lindb.: ubikv-Brezovci.

Lepidozia reptans (L.) Dumort.: bor-temp - Črni vrh; Smrekovec; ob Vrtačnikovem potoku; pod Brzovnikom.

Lophozia sudetica (Nees ex Hueben.) Grolle: bormont-Smrekovec, vzh. pob.; pri Kugovniku.

Lophozia ventricosa (Dicks.) Dumort. var. ventricosa: bor-mont - Kramarica; Brezovci.

Marchantia polymorpha L. subsp. polymorpha: bortemp-pri Kugovniku.

Marsupella emarginata (Ehrh.) Dumort. var. emarginata: bor-temp-Smrekovec, vzh. pob.; pri Kugovniku; Komen; Veliki Travnik; Presečnikov vrh.

Metzgeria conjugata Lindb.: bor-temp - Smrekovec, sev. pob.; ob Vrtačnikovem potoku; pod Pistotnikom.

Metzgeria furcata (L.) Dumort.: bor-temp - pod Brzovnikom.

Nardia scalaris Gray: bor-temp-Smrekovec; Smrekovec, vzh. pob.; Krnes; Komen.

Nowellia curvifolia (Dicks.) Mitt.: bor-temp - Smrekovec, sev. pob.; ob Vrtačnikovem potoku; pod Brzovnikom.

Odontoschisma denudatum (Mart.) Dumort.: bor-temp - Krnes, juž. pob.; pod Brzovnikom.

Pellia endiviifolia (Dicks.) Dumort.: merid-temp - Črni vrh; Smrekovec; pri Kugovniku; Vrtačnikov potok; Krnes, juž. pob.; pod Petelinjekom. 
Pellia epiphylla (L.) Corda: bor-temp - Črni vrh; Kramarica; Komen.

Pellia neesiana (Gottsche) Limpr.: bor-mont - Črni vrh; ob Vrtačnikovem potoku.

Plagiochila asplenioides (L. em Taylor) Dumort.: temp - ob Vrtačnikovem potoku; pod Brzovnikom.

Plagiochila porelloides (Torrey ex Nees) Lindenb.: subbor - Kramarica; Smrekovec, vzh. pob.; Krnes, juž. pob.; pod Brzovnikom.

Preissia quadrata (Scop.) Nees: bor-mont-Smrekovec, sev. pob.; Krnes.

Ptilidium pulcherrimum (Web.) Vanio: bor-mont Smrekovec; Kramarica.

Radula complanata (L.) Dumort.: bor-temp - ob Vrtačnikovem potoku; Krnes, juž. pob.; pod Pistotnikom.

**Riccardia chamaedryfolia (With.) Grolle: bor-temp - Smrekovec; ob Vrtačnikovem potoku;

Riccardia multifida (L.) Gray: bor-temp-Brezovci.

Riccardia palmata (Hedw.) Cerruth.: bor-mont - ob Vrtačnikovem potoku; Kolarica (Kutnar et al. 2006).

Scapania aequiloba (Schwaegr.) Dumort.: bor-mont - ob Vrtačnikovem potoku; pod Pistotnikom; Presečnikov vrh.

Scapania nemorea (L.) Grolle: bor-temp- Kramarica; Brezovci; ob Vrtačnikovem potoku; Presečnikov vrh.

Scapania umbrosa (Schrad.) Dumort.: bor-mont - ob Vrtačnikovem potoku; Krnes, juž. pob.

*Scapania undulata (L.) Dumort.: bor-temp - Smrekovec; pri Kugovniku; ob Vrtačnikovem poto$\mathrm{ku}$.

*Scapania verrucosa Heeg: temp-kont - Krnes, juž. pob.

Tritomaria execta (Schrad.) Loeske: bor-mont - pod Brzovnikom.

Tritomaria quinquedentata (Huds.) H. Buch subsp. quinquedentata: bor-mont - Kramarica; Brezovci; Komen.

\section{Horološka analiza}

Podlaga za opredelitev geoelementov in pripadnosti posamezne vrste določenemu geoelementu je delo Düll et al. (1999), vendar je klasifikacija v mnogočem modificirana z upoštevanjem del Hill \& Preston (1998) ter Martinčič (1966, 2006). Razporeditev ugotovljenih vrst $\mathrm{v}$ posamezne geoelemente je prikazana na sliki 2. Številčno najmočnejšo skupino predstavljajo borealno-temperatne vrste (sen-

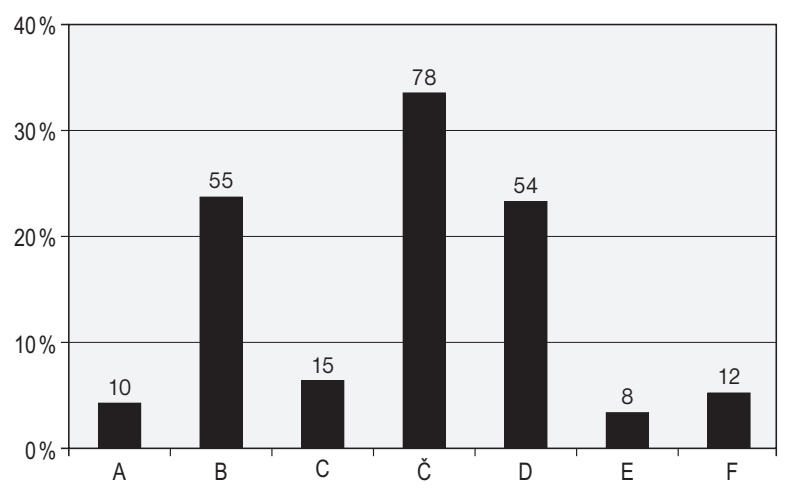

Slika 2: Razporeditev geoelementov v mahovni flori Smrekovškega pogorja, v odstotkih. Številke nad stolpci - število vrst.

A: arktično-alpinski (subarktično-subalpinski); B: borealno-montanski; C: subborealni; Č: borealno-temperatni; D: temperatni, temperatno-subkontinentalni, temperatno-subatlantski, subtemperatni; E: meridionalno-temperatni; F: druge.

Figure 2: Distribution of bryophytes of the Smrekovško pogorje Mts. over chorological elements (in \%). Numbers above bars - number of species.

A: arctic-alpine (subarctic-subalpine); B: boreal-montane; C: subboreal; Č: boreal-temperate; D: temperate, temperate-subcontinental, temperate-subatlantic, subtemperate; E: meridional-temperate; F: others.

su Hill \& Preston 1998), torej take, ki uspevajo v borealnem in temperatnem zonobiomu oziroma $\mathrm{v}$ gozdnih območjih Evrope, ki spadajo v fitogeografskem pogledu v borealno in srednjeevropsko florno regijo. Obsegajo kar 78 vrst $(33,6 \%)$ in so najbolj pogoste, z največjo pokrovnostjo. Hladnoljubnejši del mahovne flore predstavljajo arktično-alpinske (subarktično-subalpinske), borealno-montanske in subborealne vrste. Skupaj obsegajo 80 vrst $(34,4$ $\%)$. Pravi arktično-alpinski element je samo Philonotis tomentella, vse druge so subarktično-subalpinske. Njihovo število je majhno, razlog za to pa je relativno majhna nadmorska višina in manjši obseg odprtih skalnatih biotopov. Celotno Smrekovško pogorje, morda z izjemo Komna, je namreč pod gozdno mejo, ne presega montanskega pasu. Glavnino hladnoljubnejše flore tvorijo zato borealno-montanske vrste. Ta skupina obsega 55 vrst $(23,7 \%)$, kar je zelo visok delež v primerjavi $\mathrm{z}$ apnenčastimi predeli. Kisla andezitna podlaga, velika vlažnost in naravni smrekovi sestoji omogočajo tem hladnoljubnim vrstam optimalne pogoje za uspevanje. Ta »skandinavsko-alpski videz« izpopolnjujejo še subborealne vrste. Nekoliko slabše so zastopane vrste, ki jih uvr- 
ščamo v skupino temperatni element (54 vrst, 23,3 $\%$ ), ki je sicer osnovni geoelement srednjeevropske florne regije oz. temperatnega zonobioma. Glavni razlog je v tem, da se prične Smrekovško pogorje s spodnjim montanskim pasom, nad $700 \mathrm{~m}$ nadmorske višine, pomembno vpliva pa tudi hladnejša in zakisana andezitna podlaga na kateri smreka bolje uspeva kot bukev. Glavnino tvorijo splošno razširjene temperatne vrste (37 vrst, 15,9\%). Temperatno-subkontinentalni (=srednjevropski) element obsega 9 vrst $(3,8 \%)$ - to so vrste, ki v Evropi na zahodu ne dosegajo atlantskih predelov. Preostalih 8 vrst so subtemperatne in temperatno-subatlantske. Najbolj termofilno skupino predstavljajo meridionalno-temperatne vrste (south-temperate sensu Hill \& Preston 1998). To so vrste, katerih areal sega še v submediteran, najbolj pogoste pa so na toplih, kamnitih karbonatnih rastiščih. Zato je njihova maloštevilnost v Smrekovškem pogorju povsem razumljiva. V skupini »druge« so ubikvisti, po vsej Evropi splošno razširjene vrste.

\section{Ogroženost mahovne flore}

Ogroženost mahovne flore v Smrekovškem pogorju je v sedanjem času razmeroma majhna. Dosedanji vpliv človeka se je doslej omejeval pretežno na gradnjo gozdnih cest, gozdarjenje in izsekavanje gozda za pašne površine. Vpliv turizma je zaenkrat bolj viden le v predelu okrog planinskega doma. Način gospodarjenja z gozdom ohranja dovolj lesne podlage za naseljevanje lignikolnih vrst, predvsem jetrenjakov. Tudi gradnja prometnic je bila $\mathrm{v}$ določeni meri celo ugodna, saj predstavljajo cestne brežine, zlasti na skalnati podlagi, ugoden sekundarni biotop za naselitev mahovnih vrst. Izsekavanja za pašne površine že dalj časa ni več. Paše je vse manj, zato so se pričele traviščne površine zaraščati. To pa bi lahko v prihodnosti neugodno vplivalo na ohranitev močvirnih površin in mlak, zlasti na $\mathrm{Kr}$ nesu, in posledično na bogato mahovno floro tega habitata.

$\mathrm{Na}$ podlagi opredelitev $\mathrm{v}$ Rdečem seznamu (Martinčič 1992, 1996) uspeva na Smrekovškem pogorju ena prizadeta vrsta (Sphagnum compactum), 12 vrst iz kategorije »ranljive vrste « (v seznamu označene $\mathrm{z} *$ ) ter 13 vrst iz kategorije »redke vrste « (v seznamu označene $\mathrm{z} * *$ ). 4 vrste so vključene $\mathrm{v}$ evropski seznam ogroženih mahovnih vrst (ECCB 1995) in sicer v kategorijo redke (R): Scapania verrucosa, Brachythecium geheebii, Fissidens adianthoides in Paraleucobryum sauteri.

\section{SUMMARY}

The bryophyte flora of Smrekovško pogorje Mts. (The Kamniško-Savinjske Alps, Slovenia)

\section{Introduction}

The Smrekovško pogorje Mts. are located in the easternmost part of the Kamniško-Savinjske Alps. They have a characteristic ridge-like shape and stretch in an east-west direction. In the east they begin with the peak of Smrekovec (1577 m) and end with the peak of Presečnikov vrh $(1573 \mathrm{~m})$ in the west. The average altitude is $1550 \mathrm{~m}$. Hlipovec saddle betweeen Komen (1684 m) and Veliki Travnik $(1637 \mathrm{~m})$ is the exception, because the ridge descends to $1453 \mathrm{~m}$ there. The mountains are of volcanic origin and are the highest silicate complex in Slovenia. They are mainly composed of andesite and andesitic tuff, but there is also some tonalite on the northern slope. The largest part of the Smrekovško pogorje Mts.is covered with natural spruce forest (Luzulo sylvaticae-Piceetum M. Wraber 1963). However, large surfaces of this forest have been ruined by extensive pasture. On these surfaces has developed a grassland community Homogyno alpinae-Nardetum Mráz 1956. Larger beech stands (Luzulo-Fagetum Meusel 1937) grow up to the altitude of 1200-1300 m. Also worth mentioning are the stands of green alder (Alnetum viridis Br.-Bl. 1918). Springs, puddles and marshy surfaces are relatively common there because of the impermeable andesitic bedrock.

\section{Localities}

The entire range of the Smrekovško pogorje Mts. is located in two quadrants of the Central-European flora mapping (Fig. 1), namely in 9555/3 and 9554/4 (UTM network: VM94, VM84; the grid of $10 \times 10 \mathrm{~km})$. The list of species includes the abbreviated names of localities where the bryophyte material was collected.

\section{Methods}

Between 1987 and 2007 the author collected extensive moss material from the entire region of the Smrekovško pogorje. This material gives a quite perfect floristic and phytogeographic presentation of the parts reaching from $1000 \mathrm{~m}$ a.s.l. all the way 
up to the peaks. The bryophytes were collected in all of the important habitats from this region, in forests, grasslands, rocks, on marshy spots and in springs. Another important habitat, especially in forests, are also road banks. When gathering the material, all substrata, forest and grassland soil, rocks, decaying wood and tree bark were considered.

In order to present the complete bryophyte flora of the region, the data from the literature are included as well. Nomenclature and taxonomy of the species follows Schumacker \& Váňa (2005) for liverworts (Marchantiophyta) and Hill et al. (2006) for mosses (Bryophyta).

\section{Results}

The bryophyte flora from the Smrekovško pogorje Mts. is relatively unknown. So far, a more comprehensive account has been contributed only by Wallnöfer (1888) in which he published 60 species of mosses located mostly on Smrekovec. Some of the data came from M. Wraber (1963 - det. S. Grom), Piskernik (1977 - det. A. Martinčič) and Kutnar et al. (2006 - det. A. Martinčič) in their phytosociological articles. The author collected this bryophyte material in the period between 1987 and 2007. It is kept in the herbarium of the Biology Department at the Biotechnical Faculty of the University of Ljubljana (LJU).

The bryophyte flora of the Smrekovško pogorje Mts. comprises 229 species, 173 of which are mosses (Bryophyta) and 56 are liverworts (Marchantiophy$t a)$. For mosses, this number represents $28 \%$ of the currently recorded species in Slovenia (Martinčič 2003) and $43 \%$ of the species in the region of the Kamniško-Savinjske Alps, a part of which are also the Smrekovško pogorje Mts. The number of liverworts represents as much as $35 \%$ of the currently known species in Slovenia (Sabovljević \& Natcheva 2006, Ros et al. 2007). These numbers demonstrate how diverse the bryophyte flora of the study area is. The largest part of the species is recorded in the quadrant $9555 / 3$, i.e. 164 mosses and 53 liverworts. This places it among the 3 quadrants with the richest bryophyte flora in Slovenia (quadrant 9547/4 - 248 species, 0049/1 - 231 species). Three species are reported for the first time from Slovenia: Sciurohypnum oedipodium, Calypogeia integristipula and Lophozia sudetica. For Kamniško-Savinjske Alps, a part of Alpine phytogeographical region of Slovenia, the new records are also Chiloscyphus coadunatus var. coadunatus, Jamesoniella autumnalis, Jungermannia obovata, Marchantia polymorpha subsp. polymorpha, Ptilidium pulcherrimum among liverworts and Brachythecium geheebii, B. mildeanum, Bryum schleicheri var. latifolium, Grimmia trichophylla, Hygrohypnum duriusculum, Hypnum andoi, Mnium lycopodioides, Mnium spinulosum, Plagiothecium succulentum, Racomitrium affine, Sphagnum russowii among mosses.

Lophozia sudetica - Düll et al. (1999) were the first to mention a taxon with this name for Slovenia. This information was later resumed by Söderström et al. (2002), Sabovljević \& Natcheva (2006) and Ros et al. (2007). However, the first citation is based on a wrong interpretation of synonymics. There are no data on this name in primary literature (Loitlesberger 1905, Breidler 1894, Robič 1893, Glowacki 1908, 1910, 1912, 1913, Grom 1969), only a mention of the species Jungermannia muelleri Nees, Lophozia muelleri (Nees) Dum., Jungermannia alpestris Schleich. and Lophozia alpestris (Schleich.) A. Evans. Despite some confusion that is still going on regarding the nomenclature and taxonomy, it has been positively established that the listed taxa have no nomenclatural connection with the species Lophozia sudetica. The mistake therefore originates in Düll et al. (1999), which is confirmed by the added synonym L. alpestris (Schleich.) Ev. The species L. sudetica (Nees ex Huebener) Grolle (syn: L. alpestris auct. non (Schleich.) A. Evans) is therefores a new species for the Slovenian liverwort flora, with two localities in the Smrekovško pogorje Mts.

Calypogeia integristipula - a boreal-temperate species whose main distribution area is in the northern part of Europe, but which becomes more and more rare towards the south. In the Balkan peninsula it is known only from Serbia (Sabovljević \& Natcheva 2006, Ros et al. 2007). Localities in Slovenia are part of the wider Central-European part of its distribution area.

Sciuro-hypnum oedipodium - a temperate element, scattered from south Scandinavia across Central Europe, but absent from the Atlantic-Mediterranean regions. It is often overlooked because of its similarity with B. rutabulum and B. starkei. This is probably the reason why it does not occur to the south of Slovenia before the lonely locality in Prokletije (Martinčič 2006). Now it is known also from Romania and Greece (Sabovljević et al. 2008, in press). In the Smrekovško pogorje Mts. it was found only on two localities. 


\section{Chorological analysis}

The results of the chorological analysis are presented in Figure 2. The largest group comprises boreal-temperate species (sensu Hill \& Preston 1998), i.e. the species growing in the boreal and temperate zonobiome or in the wooded regions of Europe which phytogeographically belong into the boreal and Central-European floral region. They comprise as many as 76 species $(33.6 \%)$ and are the most common, with the highest cover. A more frigidophilous part of the moss flora comprises arctic-alpine (subarctic-subalpine), boreal-montane and subboreal species. Altogether they comprise 80 species $(34.4 \%)$. Philonotis tomentella is the only arctic-alpine element, all other species are subarctic-subalpine. They are small in number, because of the relatively low altitude and a smaller range of open rock biotopes. The entire Smrekovško pogorje Mts., perhaps with the exception of Komen peak, is below timberline and does not exceed the montane belt. The main part of the frigidophilous flora are therefore boreal-montane species. This group comprises 54 species $(23.7 \%)$, which is a very high proportion in comparison with the limestone areas. Acid andesite bedrock, considerable humidity and natural spruce stands provide these frigidophilous species with optimal growth conditions. This »Scandinavian-Alpine appearance « is further complemented by subboreal species. Slightly fewer in number are the species classified into the temperate element group (54 species, $23.3 \%$ ), which is a basic geoelement of the Central-European floral region or temperate zonobiome. The main reason for this is that the Smrekovško pogorje Mts. start in the lower montane belt, at about $700 \mathrm{~m}$ a.s.l. Another important factor is the colder and acid andesite bedrock which favours spruce instead of beech. The most numerous are the widely distributed temperate species (37 species, $15.9 \%$ ). The temperate-subcontinental (= Central-European) element comprises 9 species $(3.8 \%)$ - those which do not reach the Atlantic regions in the west of Europe. The remaining 8 species are subtemperate and temperate-subatlantic. The most thermophilous group are the meridional-temperate species (south-temperate sensu Hill \& Preston 1998). These are the species whose distribution area reaches into the sub-Mediterranean and which most frequently occur on warm, stony calcareous sites. Their scarcity in the Smrekovško pogorje Mts. is therefore understandable. In the »others « group are the ubiquists, the species widely distributed across Europe.

\section{Threatened taxa}

When taking into consideration the Red list for Slovenia (Martinčič 1992, 1996) we ascertain that 26 species from the list of the bryophyte flora of Smrekovško pogorje Mts. are considered threatened. Sphagnum compactum being in category »endangered «, 12 are included in category »vulnerable« (marked with * on the List of taxa) and 13 into category "rare (marked with ** on the List of taxa). Four species are included in the Red Data Book of European Bryophytes (ECCB 1995) in the rare (R) category: Scapania verrucosa, Brachythecium geheebii, Fissidens adianthoides and Paraleucobryum sauteri.

\section{REFERENCES}

Breidler, J. 1894: Die Lebermoose Steiermarks. Mitt. Naturwiss.Ver. f. Steierm. Jahrgang 1894.

Damsholt, K. 2002: Illustrated Flora of Nordic Liverworts and Hornworts. Nordic Bryological Society, Lund, 837 pp.

Düll, R, Pavletić Z. \& Martinčič, A. 1999: Checklist of the Yugoslavian bryophytes. In Düll, R., Ganeva, A., Martinčič, A. \& Pavletić, A.: Contributions to the bryoflora of former Yugoslavia and Bulgaria. IDH-Verlag Bad Münstereifel, $110 \mathrm{pp}$.

ECCB, European Committe for the Conservation of Bryophytes 1995: Red Data Book of European Bryophytes. Trondheim, 291 pp.

Glowacki, J. 1908: Die Moosflora des Bachergebirges. Jahresber. d. Obergymn. Marburg, 1-30.

Glowacki, J. 1910: Die Moosflora der Julischen Alpen. Verhandlungen der Zoologisch-Botanischen Gesellschaft in Wien 5 (2): 1-48.

Glowacki, J. 1912: Moosflora der Steiner Alpen. Jahrb. naturh. Mus. f. Kärnten, Klagenfurt »Carinthia II « Nr. 1-6:13-47; 130-156.

Glowacki, J. 1913: Ein Beitrag zur Kenntnis der Moosflora der Karstländer. Izvestja muz. društva za Kranjsko «Carniola» nov. ser. 4: 114-153.

Grolle, R. \& Long, D. G. 2000: An annotated checklist of the Hepaticae and Anthocerotae of Europe and Macaronesia. Journal of Bryology 22 (2): 103-140.

Grom, S. 1969: Mahovna flora Trnovskega gozda. Varstvo narave 6: 51-72.

Hill, M. O. \& Preston, C. D. 1998: The geographical relationships of British and Irish bryophytes. Journal of Bryology 20: 127-226. 
Hill, M. O. \& Bell, N., Bruggeman-Nannenga, M. A., Brugués, M., Cano, M. J., Enroth, J., Flatberg, K. I., Frahm, J. P., Gallego, M.T., Garilleti, R., Guerra, J., Hedenäs, L., Holyoak, D. T., Hyvönen, Ignatov, M. S., Lara, F., Mazimpaka, V., Muñoz, J., Söderström, L. 2006: An annotated checklist of the mosses of Europe and Macaronesia. Journal of Bryology 28 (3): 198-267. (DOI: 10.1179/174328206X119998)

Kutnar, L., Urbančič, M. \& Čas, M. 2006: Ohranjenost gozdnih tal in vegetacije $\mathrm{v}$ habitatu divjega petelina v vzhodnih Karavankah in vzhodnih Kamniško-Savinjskih Alpah. Zbornik gozdarstva in lesarstva 77: 5-42.

Loitlesberger, K. 1905: Zur Moosflora der österreichischen Küstenländer I. Hepaticae. Verhandlungen der Zoologisch-Botanischen Gesellschaft in Wien 55: 475-489.

Martinčič, A. 1966: Elementi mahovne flore Jugoslavije ter njihova ekološka in horološka problematika. Razprave IV. razreda SAZU 9: 5-82.

Martinčič, A. 1977: Prispevek k floristiki mahov (Bryophyta) v Sloveniji II. Biološki vestnik 25 (1): 5-14.

Martinčič, A. 1992: Rdeči seznam ogroženih listnatih mahov (Musci) v Sloveniji. Varstvo narave 8: 7-166.

Martinčič, A. 1996: Mahovi (Bryophyta). Zbornik Narava Slovenije. Stanje in perspektive: 169_ 178.

Martinčič, A. 2003: Seznam listnatih mahov (Bryopsida) Slovenije. Hacquetia 2 (1): 91-166.

Martinčič, A. 2006: Moss flora of the Prokletije mountains (Serbia, Montenegro). Hacquetia 5 (1): 113-130.

Petkovšek, V. 1945: Na savinjskih ognjenikih. Planinski zbornik: 194-203.

Piskernik, M. 1977: Gozdna vegetacija Slovenije v okviru Evropskih gozdov. Zbornik gozdarstva in lesarstva 15: 1-236.
Robič, S. 1893: Kranjski mahovi. Izvestja muzejskega društva za Kranjsko 3: 28-33, 67-75, 109-114, 148-152, 201-204.

Ros, R. M., Mazimpaka, V., Abou-Salama, U., Aleffi, M., Blockeel, T. L., Brugues, M., Cano, M. J., Cros, R. M., Dia, M. G., Dirkse, G. M., El-Saadawi, W./ Erdag, A., Ganeva, A., Gonzales-Mancebo, J. M., Herrnstadt, I., Khalil, K., Kurschner, H., Lanfranco, E., Losada-Lima, A., Refai, M. S., Rodriguez-Nunez, S., Sabovljevic, M., Sergio, C., Shabbara, H. M., Sim-Sim, M., Soderstrom, L. 2007: Hepatics and Anthocerotes of the Mediterranean, an annotated checklist. Cryptogamie Bryologie 28 (4): 351-437.

Sabovljević, M. \& Natcheva, R. 2006: A check-list of the liverworts and hornworts of Southeast Europe. Phytologia Balcanica 12 (2): 169-180.

Sabovljević, M. et al. 2008: Check-list of the mosses of Southeast Europe. Phytologia Balcanica in press.

Schumacker, R. \& Ván̆a, J. 2005: Identification keys to the liverworts and hornworts of Europe and Macronesia. 2. ed. Pozna 2005, 269 pp.

Söderström, L., Urmi, E. \& Váňa, J. 2002: Distribution of Hepaticae and Anthocerotae in Europe and Macaronesia. Lindbergia 27 (1): 3-47.

Škornik, S., Lončar, M. \& Kaligarič, M. 2006: Vegetation of silicicolous grasslands of the highlands of north-eastern Slovenia. Hacquetia 5 (1): 193-211.

Wallnöfer, A. 1888: Die Laubmoose Kärntens. Jahrb. des naturhist. Landesmus. von Kärnten 20: 1-155.

Wraber, M. 1963: Gozdna združba smreke in gozdne bekice v slovenskih vzhodnih Alpah. Razprave IV. razreda SAZU 7: 79-175.

Wraber, T. \& Skoberne, P. 1989: Rdeči seznam ogroženih praprotnic in semenk SR Slovenije. Varstvo narave 14-15: 9-428. 